\title{
OS POSSÍVEIS IMPACTOS COM O USO DA NANOTECNOLOGIA
}

\section{THE POSSIBLE IMPACTS OF USING NANOTECHNOLOGY}

\author{
Lucas Dallmann Almeida1. \\ ${ }^{1}$ Engenharia Química no Centro Universitário das Américas - FAM, São Paulo, SP.
}

Resumo. A incerteza dos perigos e riscos associados ao incremento de uma nova tecnologia, por si só já desperta o interesse e preocupação do meio acadêmico e científico; a cada ano centenas de novos produtos químicos são manipulados, incrementando qualidade de vida, ganhos, tecnologia, saúde e bem estar; mas, os riscos devem ser considerados para evitar danos ao meio ambiente, garantindo assim à sustentabilidade com a qualidade de vida e saúde do entorno impactado com a nova tecnologia. A nanotecnologia chegou, e com ela veio perspectiva de um futuro mais desenvolvido e viável para humanidade. Revolução industrial e nanoprodutos são os fatores que, para os mais otimistas, serão as estruturas básicas de um futuro próximo. Com o desenvolvimento da pesquisa, veio à tona as consequências da mesma para com o meio ambiente e a saúde. Este projeto de pesquisa apresenta alguns possíveis riscos da nanotecnologia no meio ambiente, relacionando também com a saúde de vida dos seres vivos. A partir de referências bibliográficas, este projeto foi redigido para que fique simples o entendimento nessa área.

Palavras-chave. nanotecnologia, nanopartículas, nanotoxicologia, meio ambiente, saúde

\begin{abstract}
The uncertainty of the dangers and risks associated with the increase of a new technology, on its own already arouses the interest and concern of the academic and scientific environment; every year hundreds of new chemicals are manipulated, increasing quality of life, earnings, technology, health and well-being; but, the risks must be considered to avoid damage to the environment, thus ensuring sustainability with the quality of life and health of the environment impacted by the new technology. Nanotechnology has arrived, and with it came the prospect of a more developed and viable future for humanity. Industrial revolution and nanoproducts are the factors that, for the most optimistic, will be the basic structures of a near future. With the development of research, arose the consequences for the environment and health. This research project presents some possible risks of nanotechnology in the environment, also relating to the life health of living beings. Based on bibliographical references, this project was written so that this area can be easily understood.
\end{abstract}

Keywords. nanotechnology, nanoparticles, nanotoxicology, environment, health.

Introdução. Nanotecnologia é a técnica de manipulação da matéria através dos átomos (1). O prefixo "nano" significa uma escala de um bilionésimo de metro (1,0-x10-9) (Figura 1), a palavra "tecnologia" significa um estudo sobre técnicas. (2) afirma que: segundo U.S National 
Nanotechnology Initiative (NNI), a capacidade de estudar, compreender e sintetizar partículas em escala de um e 100 nanômetros, que possibilita novas utilidades para esses materiais denominase nanotecnologia. Segundo (3), o uso dessa técnica tem dois principais objetivos:

1. Trazer mais eficiência na produção: Novos materiais eletrônicos; novos fármacos; produtos inteligentes, etc.

2. Melhorar a utilização dos materiais já criados: A nanotecnologia pode reestruturar o produto para que tenha mais utilidade ou transforma-los para atenderem outros objetivos, como a incorporação de nanopartículas em pneus, utilizado para aumentar a vida útil dos mesmos.

\section{Escala nanométrica}

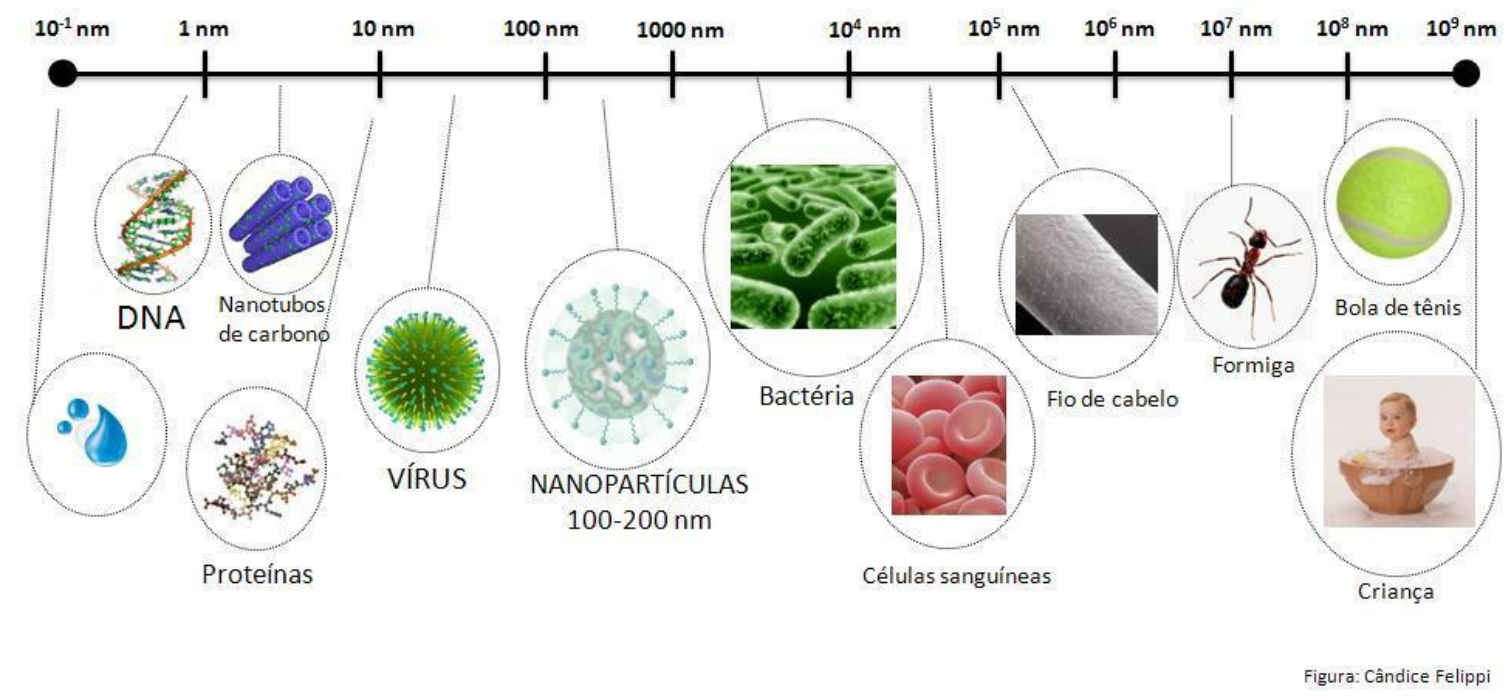

Figura 1 - Abordagem da escala manométrica para diferentes organismos (4).

Richard Feynman (1918-1988), físico americano, foi o primeiro a idealizar a técnica de manipulação da matéria em escala atômica, em 1959, e embora não tenha introduzido a palavra nanotecnologia, pode-se dizer que foi o precursor dessa área. O termo "nanotecnologia" foi utilizado, inicialmente, em 1974, pelo pesquisador da Universidade de Tóquio, Norio Taniguchi (1912-1999) com o objetivo de desenvolver ferramentas para criar dispositivos cada vez menores em chips de silício (5). 
O meio ambiente pode ser afetado devido ao grande avanço da nanotecnologia, uma vez que, o tamanho das nanopartículas facilita sua dispersão na atmosfera, na água e no solo e, sua remoção torna-se praticamente impossível por técnicas de filtração, por exemplo. No entanto, a forma com a qual as nanopartículas interagem com o meio ambiente, ainda não é compreendida com clareza devido à falta de informação (6). Além disso, quanto menor uma partícula, maior a sua reatividade, podendo desenvolver, também, novas propriedades tornando-as nocivas. Desta forma, atualmente, tem-se uma nova área da ciência, "a nanotoxicologia", que visa investigar a composição, em escala nano, dos materiais e suas relações e consequências com o meio ambiente e a saúde (7).

Atualmente, não há lei específica para nanotecnologia no Brasil, porém, com a atribuição concedida pela ISO, o Comitê Técnico no 229 tem como responsabilidade publicar normas referentes à nanotecnologia. (27). Sem possuir uma lei mundial, cada país tem sua própria normativa, podendo ser geral (normativas que analisam produtos convencionais como cosméticos, produtos químicos e medicamentos) ou específica; por exemplo, os Estados Unidos da América, que utiliza como órgão de regulamentação o Ato de Controle de Substâncias Tóxicas (TSCA), Food and Drugs Administration (FDA) e National Institute for Health (NIH) $(8,27)$.

\section{Materiais e Métodos}

Este levantamento bibliográfico foi desenvolvido a partir de estudos, pesquisas e análise de dados. Foram coletadas informações de sites, plataformas como Google Academic e SciELO, livros, artigos, revistas, dissertações, TCC (Trabalho de Conclusão de Curso), dissertação de mestrado e tese de doutorado. Participação efetiva no Seminário Internacional da Nanotecnologia, realizado na Sede da Procuradoria Regional do Trabalho da 2a . Região, em novembro de 2019. Participação no Curso de Verão do Instituto de Física da USP 2020, entre os dias 10, 11, 12, 13 e 14 de Janeiro de 2020.

\section{Fundamentação Teórica}

As nanopartículas são classificadas em naturais e sintéticas. As naturais são aquelas que sempre existiram no meio natural, porém, podem ser subdividas em acidentais e não acidentais; as acidentais dizem respeito às que decorrem de processos de interferência humana, mas não foram, intencionalmente, produzidas e, as não acidentais são aquelas que sempre existiram na natureza. As sintéticas são aquelas que, intencionalmente, foram criadas pelos humanos com objetivo de introduzi-los em algum processo (9).

Baseado no desenvolvimento, na caracterização, na produção e na aplicação de estruturas, sistemas e dispositivos com forma e tamanho na escala nanométrica, a nanotecnologia é definida como um campo científico multidisciplinar; as mesmas podem apresentar diferentes propriedades químicas, físico-químicas daquelas apresentadas, estudadas e testadas em escalas maiores (10). 
A nanotecnologia traz inúmeros investimentos e a atenção de consumidores e fornecedores, que assim, impulsionam o setor econômico; contudo, geram muitas dúvidas e preocupações para com a saúde dos seres vivos e o meio ambiente. As partículas podem ter propriedades peculiares se observadas em escala nano, porém, há riscos não estudados envolta deste assunto (1). Nessa escala tão pequena (nanômetro), os níveis de toxidade podem ser diferenciados e, possivelmente, prejudiciais ao meio ambiente e a saúde dos consumidores (11).

As embalagens e revestimentos adequados vêm ganhando grande espaço da indústria. $\mathrm{O}$ tema está se tornando cada vez mais de interesse da indústria alimentícia, que, visa melhorias como: melhorar a qualidade dos alimentos, aumentar a vida útil dos produtos e reduzir os resíduos de embalagens (12). Após a incorporação das nanopartículas nas estruturas, nanoestruturas podem ser desenvolvidas para serem capazes de mudar o produto final. Para diversos autores, algumas nanopartículas e nanoestruturas trazem os seguintes benefícios: de barreira (13);

Nanopartículas de celulose: melhorias nas propriedades mecânicas, térmicas e

- Nanosensores: têm potencial para revolucionar a precisão e a velocidade com que serão detectados os adulterantes em matrizes alimentares complexas e contaminantes moleculares pelas indústrias e agências reguladoras (14).

- Nanotubos de caborno: apresentam elevada resistência mecânica, capilaridade, estrutura eletrônica única. Com isso, o desempenho dos compósitos de nanotubos de carbono é cerca de 10 vezes melhor do que os compósitos de fibra de carbono convencionais em relação às características apresentadas (15).

- $\quad$ Nanoesfera de prata: incorporação de propriedades ativas como, por exemplo, antimicrobianas, a adição de nanoesferas de prata nas bandejas plásticas para conservação de tomates, por exemplo, (Figura 2) (16).

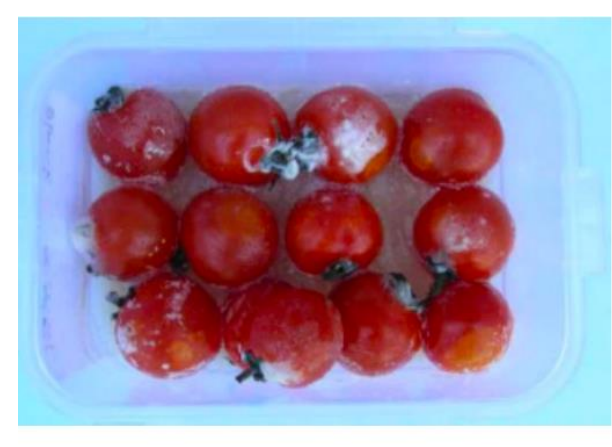

Sem nanoprata

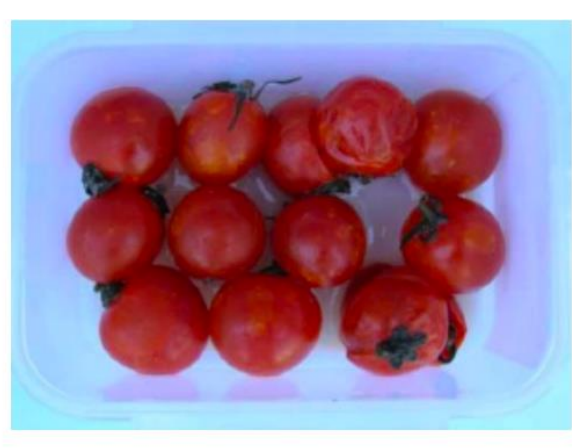

Com nanoprata

Figura 2 - Bandejas plásticas sem e com nanopartículas de prata na conservação de tomates (16).

Em contra partida, as mesmas nanopartículas/nanoestruturas podem causar impactos nocivos para com o meio ambiente. Um estudo realizado por (17) mostra que as nanopartículas de prata (NPAg), na concentração de $4,70 \mu \mathrm{g} \mathrm{L} \mathrm{L}^{-1}$, causou imobilidade de $50 \%$ dos organismos expostos ao experimento (Daphnia similis) durante 48 horas; e que, umas das maiores 
preocupações é o descarte das mesmas. Um estudo relacionado à $R$. subcapitata e T. pallida, que foram expostas à nanotubos de carbono de paredes múltiplas $(M W C N T)$ e nanotubos de carbono de paredes múltiplas funcionalizado com PEG (MWCNT-PEG) durante 72 hora, apresentou negativo para toxicidade aquática, de ambos compostos (MWCNT e MWCNT-PEG), em $R$. subcapitata, com exceção a concentração de 100 ppm (partes por milhão) para MWCNT-PEG. Mostrando-se mais sensível, a T. pallida apresentou toxicidade a partir de $10 \mathrm{ppm}$. As duas técnicas de espectroscopia utilizadas foram a Microscopia Eletrônica de Transmissão (MET), que mostrou a internalização dos nanotubos de carbono (NTC) por células de $R$. subcapitata, que indica a interação física e molecular; e a Raman confirmando que, após a exposição ao $M W C N T$ $P E G$, a célula de algas sofreram fortes alterações moleculares. (18).

A preocupação ambiental se tornou um dos principais ativismos no Brasil e no mundo nas últimas décadas. Muitos pesquisadores alertam sobre as possíveis consequências que as novas tecnologias podem trazer para o meio ambiental, pois com nossas decisões, no passado, sobre energia atômica e, atualmente, com a utilização da tecnologia genética, genética humana, nanotecnologia e ciência da informação, talvez, poderemos estar desencadeando consequências imprevisíveis, incontroláveis e, certamente até incomunicáveis, ameaçando a vida na Terra, (19). A nanotecnologia gera alta preocupação para com o meio ambiente. Quando este tema é abordado um dos pontos a serem considerados é o descarte dos nanoprodutos. As possíveis rotas de exposição das nanopartículas que são/serão descartadas no meio ambiente são o solo, sistema aquático, sedimentos e atmosfera. As principais entradas que as nanopartículas têm para com os organismos-receptores são a inalação, absorção em nível celular, ingestão e assimilação através de superfícies epiteliais externas (Figura 3) (20).

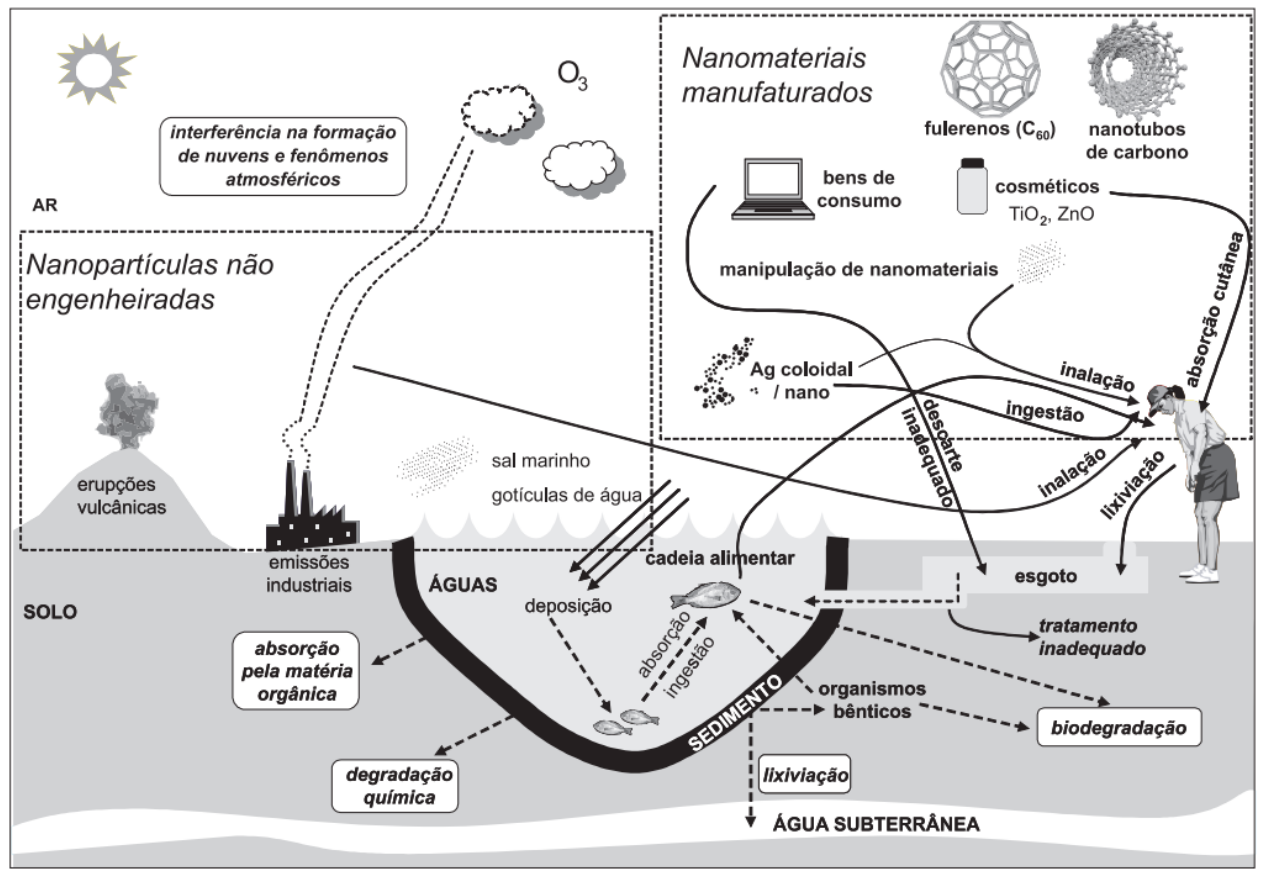


Figura 3 - Nanopartículas, em suas principais rotas, quando expostas ao meio ambiente e organismos vivos (20).

A exposição aos nanomateriais pode ocorrer por contato dérmico, ingestão e inalação e, de acordo com as características fisico-químicas do nanoproduto, este pode se depositar no solo ou ficar aderido a materiais orgânicos e inorgânicos (21). (20) mostram que as nanopartículas podem transitar a partir de portais de entradas (pele humana, pulmões e trato gastrointestinal) nos sistemas circulatórios, linfáticos e órgãos do corpo. Essas nanopartículas podem causar danos irreversíveis às células por estresse oxidativo e/ou lesão da organela.

Os materiais nanoestruturados e as nanopartículas podem acarretar em diversos problemas para saúde humana e meio ambiente. Três fatores contribuem para que esses materiais nanoestruturados e as nanopartículas possam ser tão perigosos. O primeiro é seu diminuto tamanho, que facilita sua interação com ambientes externos e fisiológicos. O segundo é o fato de que os nanomateriais podem conter metais pesados em sua composição. O terceiro é o fato de estarem sendo produzidos em escala industrial (22). As nanotecnologias são um conjunto de tecnologias, novas e revolucionárias, que, trabalhando na bilionésima parte de metro, submetem a riscos incalculáveis o consumidor, devido a suas características físico-químicas que são desconhecidas (23).

A nanotoxicologia é uma nova ciência onde tem por objetivo avaliar e estudar, em contato diretamente com o homem, a toxicidade de nanomateriais e nanodispositivos. A preocupação em torno dessa ciência deve-se ao fato de que, os nanomateriais e nanodispositivos são sintetizados, manipulados e descartados sem um rigoroso controle ou regulamentação. Contudo, a nanotoxicologia foi originada devido ao avanço das pesquisas e descobertas em volta dos possíveis impactos que a nanotecnologia (nanoprodutos, nanodispositivos, etc) podem promover ao meio ambiente. Ainda, o simples fato de partículas estarem em escala nanométrica já é motivo para os níveis de toxicidade poderem ser diferentes (11), pois quanto menor uma partícula, maior a sua reatividade.

Neste sentido, muitos pesquisadores, ou grupos de pesquisa, têm apontado que os fatores que influenciam diretamente no aumento da toxicidade das nanopartículas são o tamanho, sua solubilidade, área superficial, fator de aglomeração, forma, pureza, biodegradabilidade, composição, elevada reatividade, cristalinidade, estabilidade, via de exposição e dose administrada (9, 24, 21, 7). Por exemplo, (9) mostraram que as nanopartículas inaladas apresentam, como doenças associadas, a bronquite, asma, câncer de pulmão, doenças neurodegenerativas, tais como, Parkison e Alzheimer, além de enfisema. No trato gastrointestinal, as nanopartículas têm sido associadas à doença de Crohn e câncer de colo. Arritmia, doenças cardíacas, coágulos sanguíneos, arteriosclerose e morte cardíaca estão relacionados à nanopartículas que entram no sistema circulatório. Doenças autoimunes como doença lúpus eritematoso sistêmico, artrite e esclerodermia estão relacionados à exposição a algumas nanopartículas. A transição das nanopartículas para outros órgãos pode resultar em doenças nos mesmos. (25) mostraram que uma das características das nanopartículas é a carga (negativas, positivas ou neutras) implicando, diretamente, no sistema imunológico dos seres 
vivos. É importante lembrar que a membrana celular de um mamífero tem carga superficial negativa e, quando um cátion entra na célula, este é facilmente reconhecido como um "intruso" (devido sua diferença de composição), levando assim a ativação do sistema imunológico. Quando um aníon penetra na célula, dificilmente ele é reconhecido, pois suas propriedades são parecidas e, sendo assim, poderá levar a uma elevada taxa de toxicidade ao DNA.

(9), resumiram os possíveis efeitos adversos à saúde, associados à inalação, ingestão e contato com nanopartículas (Figura 4) e, ainda, enfatizam que nem todas as nanopartículas produzem esses mesmos efeitos adversos, uma vez que, já mencionados, a toxicidade das nanopartículas é função dos diferentes fatores, além do fato da toxicidade de uma nanopartícula qualquer, para um organismo, é determinada pelo complemento genético do indivíduo, que fornece ferramentas bioquímicas pelas quais estes podem se adaptar e combater substâncias tóxicas. 


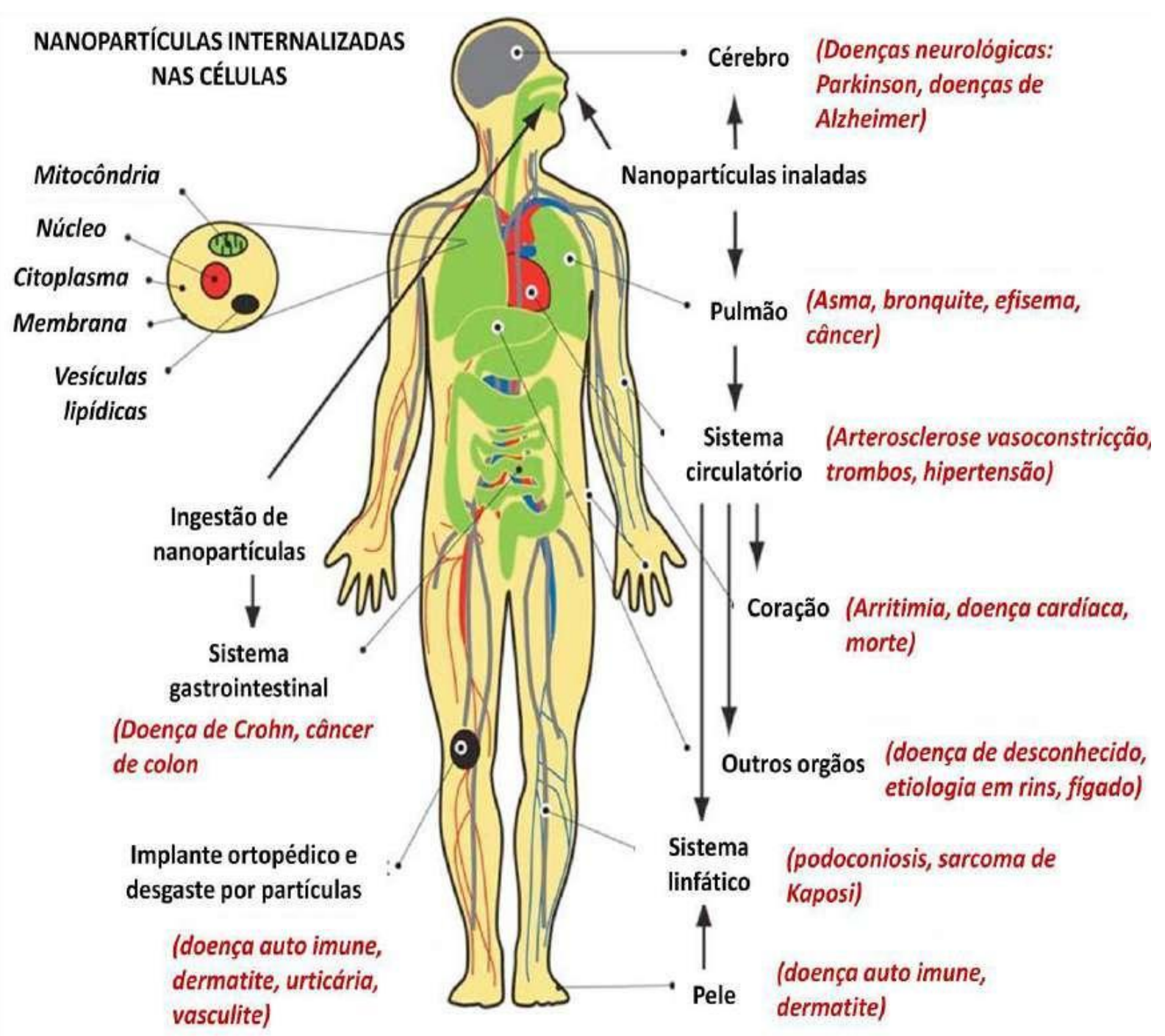

Figura 4 - Esquema do corpo humano com vias de exposição à nanopartículas, órgãos afetados e doenças associadas de estudos epidemiológicos, in vivo e in vitro (26).

\section{Conclusão.}

O fato de a nanotecnologia ser multidisciplinar, envolvendo profissionais das áreas da ciência, tecnologia e saúde, faz com que ela seja muito explorada; porém, como mostrado nesta pesquisa, há muito que pesquisar e descobrir em volta deste assunto. Segundo a plataforma Nanotechnology Produtcs Database (NPD), há, na data desta pesquisa, 8871 produtos, produzidos por 2453 empresas em 62 países, que utilizam a nanotecnologia. A indústria e o comércio usufruem da técnica para com seus produtos e os comercializam de acordo com as leis vigentes em cada país. 
O cenário mundial atual apresenta as consequências da série de erros cometidos para com o meio ambiente e a saúde humana: buracos na camada de Ozônio; extinção de espécies; radiação proveniente de explosões nucleares; etc. O domínio da nanotecnologia pode mudar o cenário mundial do comércio, contudo, o avanço da mesma está sendo realizada com normativas distintas que podem causar dúvidas em relação à procedência dos nanoprodutos. Com isso, podese afirmar que os nanoprodutos podem acarretar problemas ao meio ambiente e saúde dos seres vivos se forem expostos livremente na natureza.

\section{Referências.}

(1) Quina, F. H. NANOTECNOLOGIA E MEIO AMBIENTE: PERSPECTIVAS E RISCOS. SciELO [Internet]; 2004 [citado em 26 de novembro de 2019]. Disponível em: https://www.scielo.br/scielo.php?pid=S0100-40422004000600031\&script=sci_arttext .

(2) Lazzaretti LL, Hupffer HM. NANOTECNOLOGIA: O OLHAR DA CIÊNCIA SOBRE A TOXICIDADE E OS PONTECIAIS RISCOS DESSES PRODUTOS. Revista Conhecimento Online. Outubro de 2018; v. 3, n. 1: 79-100.

(3) Berwig JA. A GESTÃO DOS RISCOS AMBIENTAIS DA NANOTECNOLOGIA A PARTIR DE ESTUDO DE UM CASO: A Elaboração de Framework com Fundamento no Sistema do Direito Ambiental Brasileiro [dissertação de doutorado]. [São Leopoldo]: Universidade do Vale do Rio dos Sinos; 2019. 478 p.

(4) Blog UFABC Divulga Ciência [Internet]. Nanopartícula doadoras de NO. Dezembro de 2018 - [citado em 11 de julho de 2020]. Disponível em: http://proec.ufabc.edu.br/ufabcdivulgaciencia/2018/12/20/nanoparticulas-doadoras-de-no-v-1-n6-p-4-2018/ .

(5) Berger Filho AGA. GOVERNANÇA DOS RISCOS DAS NANOTECNOLOGIAS E O PRINCÍPIO DA PRECUÇÃO: UM ESTUDO A PARTIR DA TEORIA DIALÉTICA DA REDE [Dissertação de doutorado]. [São Leopoldo]: Universidade do Vale dos Sinos; 2016. 415 p.

(6) The National Institute for Occupational Safety and Health. NANOTECHNOLOGY. Centers fo Disease Control and Prevention [Internet]; 2020 [citado em 22 de junho de 2020]. Disponível em: https://www.cdc.gov/niosh/topics/nanotech/ .

(7) Ladeira RMP. Nanotoxicologia: Uma área emergente [Tese de mestrado]. [Coimbra]: Faculdade de Farmácia - Universidade de Coimbra; 2019. 59 p. 
(8) Nolasco LG, Dos Santos N. AVANÇOS NANOTECNOLÓGICOS E OS DESAFIOS REGULAMENTARES. Revista da Faculdade de Direito Universidade Federal de Minas Gerais. Dezembro de 2017; v. 71, n. 1:375-420.

(9) Buzea C, Pacheco II, Robbie K. NANOMATERIALS AND NANOPARTICLES: SOURCES AND TOXICITY. Springer [Internet]; 2007 [citado em 02 de junho de 2020]. Disponível em: https://link.springer.com/content/pdf/10.1116/1.2815690.pdf .

(10) Sant'anna LS, Alencar MSM, Ferreira AP. Patenteamento em nanotecnologia no Brasil: desenvolvimento, potencialidade e reflexões para o meio ambiente e a saúde humana. SciELO [Internet]; 2013 [citado em 20 de maio de 2020]. Disponível em: https://www.scielo.br/scielo.php?script=sci_arttext\&pid=S0100$40422013000200024 \& \operatorname{lng}=$ pt\&nrm=iso .

(11) Fernandes RG, Oliveira LPS. ENTRE RISCOS E DESINFORMAÇÃO: A UTILIZAÇÃO DA NANOTECNOLOGIA NA INDÚSTRIA DE ALIMENTOS. Revista Jurídica da FA7. Dezembro de 2019; v. 16, n. 2: 63-81.

(12) Assis LM, Zavareze ER, Prentice-Hernández C, Souza-Soares LA. REVISÃO: CARACTERÍSTICAS DE NANOPARTÍCULAS E POTENCIAIS APLICAÇÕES EM ALIMENTOS. SciELO [Internet]; 2012 [citado em 23 de junho de 2020]. Disponível em: https://www.scielo.br/scielo.php?pid=S1981-67232012005000004\&script=sci_arttext\&tlng=pt .

(13) Pereira FV, De Paula EL, De Mesquita JP, Lucas AA, Mano V. Bionanocompósitos preparados por incorporação de nanocristais de celulose em polímeros biodegradáveis por meio de evaporação de solvente, automontagem ou eletrofiação. SciELO [Internet]; 2014 [citado em 24 de junho de 2020]. Disponível em: https://www.scielo.br/scielo.php?pid=S010040422014000700017\&script=sci_arttext .

(14) Almeida ACS, Franco EAN, Peixoto FM, Pessanha KLF, Melo NR. APLICAÇÃO DE NANOTECNOLOGIA EM EMBALAGENS DE ALIMENTOS. SciELO [Internet]; 2015 [citado em 29 de maio de 2020]. Disponível em: https://www.scielo.br/scielo.php?pid=S0104$14282015000700013 \&$ script=sci_arttext\&tlng=pt\#B050 .

(15) Herbst MH, Macêdo MIF, Rocco AM. TECNOLOGIA DOS NANOTUBOS DE CARBONO: TENDÊNCIAS E PERSPECTIVAS DE UMA ÁREA MULTIDISCIPLINAR. Core [Internet]; 2004 [citado em 24 de junho de 2020]. Disponível em: https://core.ac.uk/download/pdf/204803631.pdf . 
(16) Schramm AM. INOVAÇÕES NO SISTEMA DE EMBALAGENS NAS ÁREAS DE NANOTECNOLOGIA, RADIOFREQUENCIA, DESIGN E SEGURANÇA. Instituto Mauá de Tecnologia [Internet]; 2012 [citado em 24 de junho de 2020]. Disponível em: https://maua.br/files/monografias/inovacoes-no-sistema-de-embalagens-nas-areas-

nanotecnologia-radiofrequencia-design-e-seguranca.pdf .

(17) Maziero JdaS, Rogero SO, Alemany A. Estudo ecotoxicológico da nanopartícula de prata em Daphinia similis. J Health Sci Inst. 2016;34(3):133-9.

(18) Gomes ALS, Balsam PJ, Sprogis A, Ceragioli HJ, da Silva TN, Oliveira EC, Cacur TA, Irazusta SP. AVALIAÇÃO DE TOXICIDADE DE NANOTUBOS DE CARBONO DE PAREDE MÚLTIPLA (MWCNT). Boletim Técnico da Faculdade de Tecnologia de São Paulo [Internet]; 2018 [citado em 13 de julho de 2020]. Disponível em: http://bt.fatecsp.br/media/bulletins/bt45v2.pdf\#page=15 .

(19) BECK U. INCERTEZAS FABRICADAS. Revista do Instituto Humanitas Unisinos [Internet]; 2006 [citado em 18 de maio de 2020]. Disponível em: http://www.ihuonline.unisinos.br/media/pdf/IHUOnlineEdicao181.pdf .

(20) Paschoalino MP, Marcone GPS, Jardim WF. Os nanomateriais e a questão ambiental. SciELO [Internet]; 2010 [citado em 27 de julho de 2020]. Disponível em: https://www.scielo.br/scielo.php?script=sci_arttext\&pid=S0100-40422010000200033 .

(21) Jesus KRE, Castro VLSS, Assis OBG. ANÁLISE INTEGRADA DOS CRITÉRIOS DE SEGURANÇA AMBIENTAL DOS NANOPRODUTOS. Embrapa [Internet]; 2014 [citado em 22 je de 2020]. Disponível em: https://ainfo.cnptia.embrapa.br/digital/bitstream/item/117028/1/2014AA49.pdf .

(22) Gomes RC, Pastore VAA, Martins AO, Biondi GF. APLICAÇÕES DA NANOTECNOLOGIA NA INDÚSTRIA DE ALIMENTOS. UMA REVISÃO. Revista Brasileira de Higiene e Sanidade Animal [Internet]; 2015 [citado em 21 de fevereiro de 2020]. Disponível em: http://www.higieneanimal.ufc.br/seer/index.php/higieneanimal/article/view/230 .

(23) Da SILVA LEAL, D. W.; HOHENDORFF, R. V. A era das nanotecnologias no mercado consumidor: A inserção dos "nanoprodutos" ao cotidiano e o direito à informação. Direito e Desenvolvimento, v. 9, n. 2, p. 286-302, João Pessoa, Ago. - Dez./2018.

(24) CARVALHO RV. Nanotoxicologia: segurança no uso de nanopartículas [Trabalho de Conclusão de Curso]. [Porto Alegre]: Universidade Federal do Rio Grande do Sul; 2011. 51 p. 
(25) Adabi M, Naghibzadeh M, Adabi M, Zarrinfard MA, Esnaashari SS, Seifalian AM, FaridiMajidi R, Aiyelabegan HT Ghanbari H. Biocompatibility ad Nanostructured materials: applications in nanomedicine. ResearchGate [Internet] Disponível em: <https://www.researchgate.net/publication/303721422_Biocompatibility_and_nanostructured_m aterials_applications_in_nanomedicine>. Acesso em 23/06/2020.

(26) Nolasco LG. REGULAMENTAÇÃO JURÍDICA DA NANOTECNOLOGIA [Tese de PósGraduação]. [Goiânia]: Universidade Federal de Goiás; 2016. 417 p.

(27) Lazzaretti LL, Hupffer HM. NANOTECNOLOGIA E SUA REGULAMENTAÇÃO NO BRASIL. Revista Gestão e Desenvolvimento. Julho de 2019; v. 16, n. 3: 154-177. 